\title{
Consequences of vanishing twins in IVF/ICSI pregnancies
}

\author{
Anja Pinborg ${ }^{1,3}$, Øjvind Lidegaard ${ }^{2}$, Nina la Cour Freiesleben ${ }^{1}$ and Anders Nyboe Andersen $^{1}$ \\ ${ }^{1}$ The Fertility Clinic, The Juliane Marie Center, Copenhagen University Hospital, Rigshospitalet, Blegdamsvej 9, 2100 Copenhagen and \\ ${ }^{2}$ Department of Obstetrics \& Gynecology, Herlev University Hospital, Herlev, Denmark \\ ${ }^{3}$ To whom correspondence should be addressed. E-mail: apinborg@ rh.hosp.dk
}

BACKGROUND: Spontaneous reductions are a possible cause of the increased morbidity in IVF singletons. The aim of this study was to assess incidence rates of spontaneous reductions in IVF/ICSI twin pregnancies and to compare short- and long-term morbidity in survivors of a vanishing co-twin with singletons and born twins. METHODS: We identified 642 survivors of a vanishing co-twin, 5237 singletons from single gestations and 3678 twins from twin gestations. All children originated from pregnancies detected by transvaginal sonography in gestational week 8. By crosslinkage with the national registries the main endpoints were prematurity, birth weight, neurological sequelae and mortality. RESULTS: Of all IVF singletons born, 10.4\% originated from a twin gestation in early pregnancy. Multiple logistic regression analyses adjusted for maternal age, parity and ICSI treatment showed for birth weight $<2500 \mathrm{~g}$ an odds ratio (OR) of 1.7 [95\% confidence interval (CI) 1.2-2.2] and for birth weight $<1500 \mathrm{~g}$ OR 2.1 (95\% CI 1.3-3.6) in singleton survivors of a vanishing twin versus singletons from single gestations; corresponding figures were seen for preterm birth. This increased risk was almost entirely due to reductions that occurred at $>8$ weeks gestation. We found no excess risk of neurological sequelae in survivors of a vanishing co-twin versus the singleton cohort; however, OR of cerebral palsy was 1.9 (95\% CI 0.7-5.2). Furthermore, we observed a correlation between onset of spontaneous reduction, i.e. the later in pregnancy the higher the risk of neurological sequelae $(r=-0.09 ; P=0.02)$. Adjusted OR of child death within the follow-up period was 3.6 (95\% CI 1.7-7.6) in the survivor versus the singleton cohort. CONCLUSIONS: One in $10 \mathrm{IVF}$ singletons originates from a twin gestation. Spontaneous reductions that occur at $>8$ weeks gestation are one of the causes for the higher risk of adverse obstetric outcome in IVF singletons.

Key words: Cerebral palsy/neonatal outcome/spontaneous reduction/vanishing twin

\section{Introduction}

Currently, $4 \%$ of Danish children are born as a result of IVF or ICSI (Nyboe Andersen et al., 2004). Recently, it has become evident that IVF/ICSI singletons are more likely to be born preterm and to be of low birth weight than their naturally conceived counterparts (Schieve et al., 2002; Helmerhorst et al., 2004; Jackson et al., 2004). Moreover, two studies found that IVF singletons also carry a higher risk of cerebral palsy (Strömberg et al., 2002; Lidegaard et al., 2005). The evidence relating to the causes of this adverse outcome in IVF singletons is less clear. Some researchers have argued that the excess risk may be due to the underlying infertility of the couples seeking treatment rather that the treatment itself (Pandian et al., 2001; Basso and Baird, 2003). As the transfer of two embryos or more is still the routine in most countries, a plausible explanation for the poorer outcome in IVF singletons is that a sizeable number of IVF singletons origin from twin gestations, where spontaneous reduction occurred, leaving the surviving co-twin with an increased risk of adverse outcome.

Late intrauterine death of one twin in naturally conceived twin pregnancies is associated with a considerably increased risk of morbidity and mortality in the surviving co-twin (Pharoah and Adi, 2000; Scher et al., 2002). Moreover, two previous studies on 213 and 1597 clinical assisted reproductive technique pregnancies demonstrated that $12-30 \%$ of twin pregnancies, recognized by transvaginal sonography in gestational week 8 , end up as a singleton birth, $60-83 \%$ as twin deliveries and $5-10 \%$ as spontaneous abortions of both fetuses (Landy and Keith, 1998; Tummers et al., 2003). The current knowledge on the incidence of spontaneous reductions (vanishing twins) in IVF pregnancies is scarce, and only a few studies have shown that the higher the number of gestational sacs the higher obstetric risks, irrespective of the final birth number (Dickey et al., 2002; Schieve et al., 2002; Lancaster, 2004). However, a recent study with 6377 IVF infants showed that elevated risk ratios of five perinatal outcomes of IVF singletons remained elevated although diminished, when restricted to infants with only one fetal heart in early pregnancy (Schieve et al., 2004). This indicates that vanishing twins are not the sole contributor to the adverse outcome in IVF singletons. Probably owing to its small size, an Israeli study on 426 singleton IVF births reported no effect of the starting number of embryos on either gestational age or birth weight (La Sala et al., 2004). As the literature on the influence of spontaneous reduction on short- and long-term morbidity in IVF singletons is limited, the 
aim of this study was to assess incidence rates of spontaneous reduction (vanishing twin) in a large Danish cohort of IVF singletons, and to compare obstetric outcome, neurological sequelae and mortality in survivors of a vanishing co-twin with singletons from a single gestation and born twins.

\section{Materials and methods}

The study included data on 8542 women with ongoing IVF/ICSI pregnancies detected by transvaginal sonography in gestational week 8 at 11 Danish fertility clinics, with date of embryo replacement consecutively registered between January 1, 1995 and December 31, 2001. The eight participating public fertility clinics were situated at Copenhagen University Hospital, Rigshospitalet, Herlev and Hvidovre, Odense University Hospital, Aarhus University Hospital in Skejby, Brædstrup, Skive and Holbæk Hospital, and the three private clinics were Gentofte and Odense Fertility Clinic and Ciconia Vest. According to unpublished data from the Danish Fertility Society, these 11 clinics represent $72 \%$ of all IVF and ICSI cycles performed during the study period in Denmark.

Only pregnancies that fulfilled the following inclusion criteria in gestational week 8 were eligible: (A) one fetus with positive fetal heart rate plus a gestational sac/fetus without fetal heart beat; (B) one viable fetus; or (C) two viable fetuses. Pregnancies in which more than two fetal hearts were beating and gestations with no viable fetuses were excluded. The key variables in each record of pregnancy were the date of embryo replacement and the Central Person Register number of the woman, which is provided to every individual in Denmark. Retrieval of register data was conducted in three steps. First, a cross-linkage with the National IVF Registry and the Danish Medical Birth Registry to identify the outcome of each pregnancy as a spontaneous abortion, a singleton or a twin delivery, and the number live-born and stillborn. The second step was to separate the children into the three study cohorts. Group I, the 'survivor cohort', consisted of singleton born children detected as twin gestations according to inclusion criteria $\mathrm{A}$ or $\mathrm{C}$ by sonographic diagnostics in early pregnancy. This cohort also included the live-born twin of a set of twins, where one baby was live-born and one baby stillborn (Table I). Hence, the survivor cohort comprised children from pregnancies with twin conceptions in early pregnancy, where one fetus either vanished or died in utero. Group II, the 'singleton cohort', consisted of born singletons registered with only one heart beat (inclusion criteria B) in early pregnancy. Group III, the 'twin cohort', comprised live-born twins from gestations with two fetal heart beats according to inclusion criteria $\mathrm{C}$.

Subsequently, the survivor cohort was divided with respect to the time of vanish or death of co-twin: early vanish, co-twin perished \& gestational weeks; intermediate vanish, co-twin disappeared between $>8$ and <22 gestational weeks; and late vanish, co-twin was stillborn, i.e. died $\geq 22$ gestational weeks. In Denmark, fetuses dying after $\geq 22$ gestational weeks are registered as stillborn.

We identified 8542 records on pregnancies diagnosed by transvaginal ultrasonography in gestational week 8 . Identification of pregnancy outcome was possible in 8262 (96.7\%) pregnancies and the remaining 280 (3.3\%) pregnancies were lost to follow-up, mainly due to foreign patients receiving IVF treatment in Denmark and to emigration.

In the third step of the study, an existing linkage between a mother and her children enabled us to identify children in the three cohorts. By cross-reference of the children's Central Person Register numbers with The Danish Hospital Register (Andersen et al., 1999), we identified discharge diagnosis codes recorded according to the International Classification of Diseases, 10th edition (ICD-10), on all children diagnosed or treated in a hospital setting as in- or outpatients. As some neurodevelopmental diseases might have been referred only to a psychiatric hospital, diagnoses were retrieved also from the Danish Psychiatric Central Registry (Munk-Jorgensen and Mortensen, 1997). To ensure an adequate follow-up, retrieval of diagnoses were performed until August 10, 2004, when all children were aged between 2.5 and 9 years. Infant deaths were retrieved from the Danish Registry of Causes of Deaths.

\section{Outcome measures}

The outcome measures were prevalence of spontaneous abortions $₫ 2$ or $>12$ gestational weeks, birth weight, gestational age, malformations, admissions to neonatal intensive care unit (NICU) (date of entrance $\$ 8$ days after delivery) and mortality rates. With respect to

Table I. The number of spontaneous abortions, singleton and twin deliveries, live-born and stillborn children according to the ultrasonographic diagnostics in gestational week 8

\begin{tabular}{|c|c|c|c|c|}
\hline \multirow[t]{2}{*}{ Inclusion criteria: } & \multirow{2}{*}{$\begin{array}{l}\text { One viable fetus and one gestational } \\
\text { sac/fetus without heartbeat }[n(\%)] \\
\text { A }\end{array}$} & \multirow{2}{*}{$\begin{array}{l}\text { One viable fetus }[n(\%)] \\
\text { B }\end{array}$} & \multirow{2}{*}{$\begin{array}{l}\text { Two viable fetuses }[n(\%)] \\
\text { C }\end{array}$} & \multirow[t]{2}{*}{ Total $(n)$} \\
\hline & & & & \\
\hline Spontaneous abortion week $₫ 2$ & $14(3.1)$ & $251(4.4)$ & $14(0.7)^{b}$ & 279 \\
\hline Spontaneous abortion week $>12$ & $13(2.9)$ & $131(2.3)$ & $61(2.8)^{\mathrm{b}}$ & 205 \\
\hline Spontaneous abortion unknown GA & $1(0.2)$ & $9(0.2)$ & $0(0)^{\mathrm{b}}$ & 10 \\
\hline \multicolumn{5}{|l|}{ No. of children } \\
\hline Singletons, live-born & 424 (I) & 5237 (II) & $187(\mathrm{I})$ & 5848 \\
\hline Twins, live-born & 0 & 0 & 3709 (III) & 3709 \\
\hline Total, live-born & 424 & 5237 & 3896 & 9557 \\
\hline Stillborn (per 1000 children) & $1(2.4)$ & $33(6.3)$ & $40(10.2)^{\mathrm{c}}$ & $74(7.7)$ \\
\hline Total, live-born \pm stillborn & 425 & 5270 & 3936 & 9631 \\
\hline
\end{tabular}

${ }^{\mathrm{a} T}$ Total number of pregnancies 8262 , of which 11 triplet deliveries were excluded.

${ }^{\mathrm{b}}$ Abortion of both twins in a twin set.

${ }^{\mathrm{c}}$ One of the 40 stillborn was a singleton delivery, 39 from twin deliveries (31 in which one twin was stillborn, four in which both twins were stillborn).

$\mathrm{I}=$ survivor cohort: twin conception and one live-born singleton: early loss, $n=424$; loss after week $8, n=187$; and stillborn co-twin, $n=31$; in total $n=642$.

II = singleton cohort: singleton conceptions and live-born singletons; $n=5237$. III = twin cohort: two vital fetuses in week 8 and two live-born children; $n=3896-$

$187=3709-31$ stillborn co-twins $=3678$. GA-gestational age . 
neurological sequelae the three primary outcome measures were: (i) cerebral palsy, specifically DG80.0-DG83.8; (ii) neurological sequelae, defined as mental retardation (DF70.0-79.9), severe mental developmental disturbances (DF84.0-89.9), cerebral palsy (DG80.0-83.8) and retarded psychomotor development (DR62.0); and (iii) all neurodevelopmental diseases (all DF, DG and DR62.0 diagnosis codes). A ranking system of the diagnosis codes was outlined prior to data retrieval (Pinborg et al., 2004a).

Recently, we have published data on obstetric outcome and neurological sequelae in the Danish National Twin Birth Cohort 1995-2000 (Pinborg et al., 2004a; b; c), which do not contain data on early ultrasonography. In the present study, data are based on sonographic diagnostics on clinical pregnancies recorded at the fertility clinics, which are the only way to assess vanishing twins; further children born up to 2002 were included. A new cross-linkage with the national registries was performed and there have been no referral of data from the previous studies to this study, but of course as the study periods overlap, some children have been included in both the previous and the present study.

\section{Statistical analysis}

Statistical analysis was performed using SPSS for Windows, version 10.0. A $P$-value of $<0.05$ was considered statistically significant. Differences of means of continuous parametric data were analysed with the use of Student's $t$-test. $\chi^{2}$ analyses were used to compare distributions between groups. In relation to early, intermediate and late vanish of co-twin, Spearman correlation coefficient $(r)$ for ordinal data was calculated. Odds ratios (ORs) for cerebral palsy, neurological sequelae and neurodevelopmental diseases were calculated with the Mantel-Haenszel estimate after stratification for child sex and gestational age ( $<32,32-36$ and $\geq 37$ weeks).

Multiple logistic regressions were performed to determine which variables independently predicted (i) preterm birth, (ii) low birth weight and (iii) neurological sequelae. The following variables were entered as potential confounders: maternal age at delivery $(<35$ or $\geq 35$ years), parity (less than one or one or more previous deliveries), child sex, IVF versus ICSI, child age (continuous variable) and spontaneous versus no spontaneous reduction.

\section{Results}

\section{Pregnancy outcome}

Of the 8262 early live pregnancies with identified pregnancy outcome, $494(6.0 \%)$ were spontaneous abortions and 7768 (94.0\%) deliveries. Eleven deliveries resulted in triplet births and were excluded. The number of spontaneous abortions, singletons and twin deliveries according to the result of the sonographic diagnostics in gestational week 8 are reported in Table I.

A total of 7757 deliveries were identified; 5883 (75.8\%) singleton births and 1874 (24.2\%) twin births. Thus, 9631 children were included in the study: 3748 twins (38.9\%) and 5883 $(61.1 \%)$ singletons (Table I). Seventy-four children were stillborn. Thirty-five were born in a singleton delivery and 39 in a twin delivery. Eight of the stillborn twins originated from four twin pairs where both children were stillborn and 31 had a liveborn co-twin. These 31 survivors of a stillborn co-twin were included in the survivor cohort (Table I).

\section{Abortions}

The outcome of 494 pregnancies was a spontaneous abortion. The number of abortions at $₫ 2$ or $>12$ gestational weeks according to the result of the early sonography is presented in Table I. Of pregnancies originating from one fetal heart beat, $6.9 \%$ resulted in abortion and $93.1 \%$ in singleton delivery. If one fetus was lost/perished at early sonography, $6.2 \%$ resulted in spontaneous abortion and $93.8 \%$ in singleton delivery. Of those from two fetal heartbeats, only $3.5 \%$ resulted in abortion of both, $8.8 \%$ in singleton delivery and $87.7 \%$ in twin delivery. Twentysix $(5.3 \%)$ of all abortions were registered as induced abortions, the majority probably due to malformations of the fetus.

Of pregnancies with one viable fetus in week 8, almost twothirds of spontaneous abortions occurred in the first trimester $(251 / 391 ; 64.2 \%)$. In pregnancies with one fetus perished in gestational week 8 , approximately one-half resulted in spontaneous abortion before and one-half after 12 weeks of gestation. On the other hand, for gestations with two viable fetuses the vast majority of spontaneous abortions occurred in or after the second trimester $(61 / 75 ; 81.3 \%)$ (Table I).

\section{Demographic data and obstetric outcome}

The number of live-born singletons and twins according to the result of the early sonographic diagnostics is listed in Table I. The singleton cohort included singletons from singleton gestations $(n=5237)$. The survivor cohort $(n=642)$ consisted of children born as singletons after a twin conception in early pregnancy (424 were the result of early loss; 187 resulted from loss after week 8; and 31 were survivors of a stillborn co-twin. The twin cohort comprised live-born twins $(n=3678)$.

Maternal characteristics and obstetric outcomes of the children are listed in Table II. There were no significant differences between the three cohorts according to parity and child sex, however twin mother were significantly younger than mothers in the Survivor cohort. Mean birth weight and gestational age was significantly lower in survivors than in singletons $(P<0.001)$, but significantly higher than in the twin cohort $(P<0.001)$. In accordance, the proportion of children with low birth weight $(<2500 \mathrm{~g})$ and very low birth weight $(<1500 \mathrm{~g})$ was significantly higher in the survivor than the singleton cohort, whereas it was highest in the twin cohort. Similar observations were made in terms of preterm ( $<37$ weeks) and very preterm birth ( $<32$ weeks) (Table II). As shown in Table III, only mean birth weight differed significantly between the early loss group and singletons, whereas the frequencies of children born preterm or with low birth weight were equal. However, there were significant differences between loss at $>8$ weeks of gestation and the early loss group according to prematurity and low birth weight (Table III).

Similar rates of NICU admittance were observed in survivors and singletons, whereas the rate for twins was significantly higher (Table II). Survivors spent on average more days in the NICU than singletons and less days than twins. There was a near significant increased risk of spending $>7$ days in the NICU in survivors versus singletons [OR $1.495 \%$ confidence interval (CI) 1.0-1.9], but no increased risk regarding stay for $>28$ days. Similar total malformation rates were seen in survivors, singletons and twins (Table II). In the survivor cohort the proportion of children admitted to the NICU in the early, intermediate and late vanish group was $29.0 \%, 31.0 \%$ and $51.6 \%$, respectively (Table III). 
Table II. Maternal and infant characteristics in the survivor, singleton and twin cohort

\begin{tabular}{|c|c|c|c|c|c|}
\hline & Survivor cohort & Singleton cohort & $P$-values ${ }^{\mathrm{a}}$ & Twin cohort & $P$-values ${ }^{\mathrm{b}}$ \\
\hline No. of live-born children & 642 & 5237 & & 3678 & \\
\hline Boy $(\%)$ & 53.1 & 51.8 & 0.6 & 52.8 & 0.9 \\
\hline Girl (\%) & 47.9 & 48.7 & & 47.4 & \\
\hline Birth weight $(\mathrm{g})($ mean $\pm \mathrm{SD})$ & $3264 \pm 795$ & $3442 \pm 662$ & $<0.001$ & $2471 \pm 620$ & $<0.001$ \\
\hline Gestational age (weeks) (mean $\pm \mathrm{SD})$ & $38.9 \pm 3.4$ & $39 \cdot 5 \pm 2.6$ & $<0.001$ & $36.2 \pm 3.3$ & $<0.001$ \\
\hline Birth weight <2500 g $(\%)$ & 11.7 & 6.3 & $<0.001$ & 45.4 & $<0.001$ \\
\hline Birth weight $<1500 \mathrm{~g}(\%)$ & 4.1 & 1.5 & $<0.001$ & 7.7 & $<0.001$ \\
\hline Gestational age $<37$ weeks $(\%)$ & 13.2 & 9.0 & $<0.001$ & 47.1 & $<0.001$ \\
\hline Gestational age <32 weeks (\%) & 3.8 & 1.3 & $<0.001$ & 8.7 & $<0.001$ \\
\hline Malformations (\%) & 9.2 & 9.3 & 0.9 & 10.9 & 0.2 \\
\hline \multicolumn{6}{|l|}{ Mortality } \\
\hline Neonatal up to day 28 & 7 & 16 & & 32 & \\
\hline Day 29 to 1 year & 3 & 7 & & 6 & \\
\hline$>1$ year & 0 & 1 & & 3 & \\
\hline Total $[n($ per 1000$)]$ & $10(15.6)$ & $24(4.6)$ & 0.001 & $41(11.1)$ & 0.3 \\
\hline NICU admissions (\%) & 30.7 & 28.0 & 0.2 & 55.8 & $<0.001$ \\
\hline Days in NICU (mean) & 15.5 & 11.4 & 0.01 & 22.2 & $<0.001$ \\
\hline$>7$ days in NICU $(\%)$ & 46.5 & 38.5 & 0.05 & 80.1 & $<0.001$ \\
\hline$>28$ days in NICU $(\%)$ & 14.1 & 9.8 & 0.09 & 26.3 & $<0.001$ \\
\hline Maternal age at delivery, years (mean $\pm \mathrm{SD}$ ) & $33.3 \pm 3.9$ & $33.3 \pm 3.7$ & 0.7 & $32.6 \pm 3.6$ & $<0.0001$ \\
\hline Age $<30$ years $(\%)$ & 20.3 & 18.8 & 0.4 & 23.4 & 0.09 \\
\hline Nulliparity $(\%)$ & 82.7 & 81.2 & 0.4 & 81.6 & 0.5 \\
\hline \multicolumn{6}{|l|}{ Treatment method (\%) } \\
\hline IVF & 65.9 & 62.8 & 0.1 & 65.8 & 0.3 \\
\hline ICSI & 26.4 & 26.7 & & 26.8 & \\
\hline FER & 6.6 & 8.9 & & 5.4 & \\
\hline $50 \%$ IVF/50\% ICSI & 1.1 & 1.6 & & 2.0 & \\
\hline
\end{tabular}

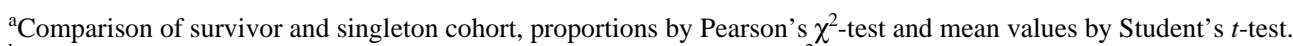

${ }^{\mathrm{b}}$ Comparison of survivor and twin cohort by proportions by Pearson's $\chi^{2}$-test and mean values by Student's $t$-test.

FER $=$ frozen embryo replacement; NICU = neonatal intensive care unit.

I'able III. Neonatal outcome in the survivor cohort according to time of vanish of co-twin

\begin{tabular}{|c|c|c|c|c|c|c|c|}
\hline & Singletons without vanish & $P$-value ${ }^{\mathrm{a}}$ & Early vanish & $P$-value ${ }^{\mathrm{b}}$ & Intermediate vanish & $P$-value ${ }^{\mathrm{c}}$ & Late vanish \\
\hline Live-born children $(n)$ & 5237 & & 424 & & 187 & & 31 \\
\hline Birth weight $(\mathrm{g})($ mean $\pm \mathrm{SD})$ & $3442 \pm 662$ & 0.02 & $3365 \pm 695$ & $<0.001$ & $3185 \pm 867$ & $<0.001$ & $2178 \pm 940$ \\
\hline Gestational age (weeks) (mean \pm SD) & $39.5 \pm 2.6$ & 0.3 & $39.4 \pm 2.6$ & $<0.001$ & $38.5 \pm 4.1$ & $<0.001$ & $34.3 \pm 4.8$ \\
\hline Birth weight $<2500 \mathrm{~g}(\%)$ & 6.3 & 0.2 & 7.9 & 0.01 & 14.4 & $<0.001$ & 56.0 \\
\hline Birth weight $<1500 \mathrm{~g}(\%)$ & 1.5 & 0.5 & 1.9 & 0.02 & 5.3 & $<0.001$ & 32.0 \\
\hline Gestational age $<37$ weeks $(\%)$ & 9.0 & 0.8 & 9.5 & 0.03 & 15.5 & $<0.001$ & 53.6 \\
\hline Gestational age $<32$ weeks (\%) & 1.3 & 0.4 & 1.9 & 0.02 & 5.3 & 0.002 & 21.4 \\
\hline \multicolumn{8}{|l|}{ Mortality } \\
\hline Neonatal up to day $28(n)$ & 16 & 0.5 & 1 & $<0.001$ & 5 & 0.8 & 1 \\
\hline Day 29 to 1 year $(n)$ & 7 & 0.7 & 0 & 0.6 & 3 & $<0.001$ & 0 \\
\hline After 1 year $(n)$ & 1 & 0.5 & 0 & 0.5 & 0 & $<0.001$ & 0 \\
\hline Total mortality [ $n$ (rate per 1000$)]$ & $24(4.6)$ & 0.7 & $1(2.4)$ & 0.2 & $8(42.8)$ & $<0.001$ & $1(32.3)$ \\
\hline \multicolumn{8}{|l|}{ NICU } \\
\hline NICU admissions (\%) & 28.0 & & 29.0 & & 31.0 & & 51.6 \\
\hline$>7$ days in NICU (\%) & 38.5 & & 41.7 & & 47.1 & & 75.0 \\
\hline$>28$ days in NICU $(\%)$ & 9.8 & & 8.7 & & 15.7 & & 43.8 \\
\hline
\end{tabular}

aCComparison of singletons versus early vanish of co-twin. Proportions by Pearson's $\chi^{2}$-test and mean values by Student's $t$-test.

${ }^{b}$ Comparison of early versus intermediate vanish of co-twin. Proportions by Pearson's $\chi^{2}$-test and mean values by Student's $t$-test.

${ }^{c}$ Comparison of intermediate versus late vanish of co-twin. Proportions by Pearson's $\chi^{2}$-test and mean values by Student's $t$-test.

Early vanish $=$ spontaneous reduction gestational week $<8$; intermediate vanish $=$ gestational week $>8$ and $<22$; late vanish $=$ recorded as stillbirth of

co-twin gestation week $>22$; NICU $=$ neonatal intensive care unit.

\section{Mortality}

Mortality rates in the three cohorts are presented in Table II. The OR of child death adjusted for maternal age (>35 years) and parity (less than one delivery) in the survivor versus the singleton cohort was 3.6 (95\% CI 1.7-7.6), and the adjusted OR of child death in the survivors versus twins was 1.4 (95\% CI 0.7-2.9). Adjusting also for gestational age, OR decreased for survivors versus singletons (OR 2.1; 95\% CI 0.9-4.6) and increased for survivors versus twins (OR 2.4; 95\% CI 1.1-5.0). As expected, the vast majority of child deaths appeared in the neonatal period. Regarding the survivor cohort, mortality rates were significantly higher in the late (32.3 per 1000) and intermediate (42.8 per 1000) vanish groups compared with the early vanish group $(2.4$ per 1000) $(P<0.001)$ (Table III). The mortality rate in the early vanish group was comparable to that of the singleton cohort (Table III). 


\section{The early and intermediate vanish cohort}

We performed separate analyses on the survivor cohort after exclusion of survivors of a stillborn co-twin $(n=31)$. Whereas children in the early and intermediate group are recorded as singletons in the birth registries, survivors of a stillborn cotwin are registered as twin births. The intermediate and early survivor group $(n=611)$ comprised $611 / 5848(10.4 \%)$ of the total number of IVF/ICSI singletons. The frequency of preterm birth was $11.3 \%$ versus $9.1 \%(P=0.07)$ and very preterm births $3.0 \%$ versus $1.3 \%(P=0.002)$ in the early and intermediate survivor versus the singleton cohort. The proportion of low birth weight was $9.9 \%$ versus $6.3 \%(P=0.001)$ and very low birth weight $3.0 \%$ versus $1.5 \%(P=0.006)$ in the early and intermediate survivor cohort versus the singleton cohort. In addition, mean gestational age 39.1 versus 39.5 weeks $(P=0.003)$ and mean birth weight $3309 \mathrm{~g}$ versus $3442 \mathrm{~g}(P<0.001)$ was significantly lower in the early and intermediate survivor versus the singleton cohort. Infant mortality rates were 14.7 per 1000 live-born children in the early and intermediate survivor cohort and 4.6 per 1000 in the singleton cohort $(P=0.002)$. The maternal age and parity-adjusted OR of child death within the follow-up period was 3.3 (95\% CI 1.6-7.3) in the early and intermediate survivor cohort versus the singleton cohort, but the risk increase disappeared after correction also for gestational age (OR 2.3; 95\% CI 1.0-5.3). Comparing only the early vanish group with the singletons, we found a significant lower mean birth weight among early survivors; however, no statistically significant differences were found for any of the other outcomes (Table III).

\section{Predictors of preterm birth and low birth weight}

To explore the predicting factors of preterm birth and low birth weight in the survivor and singleton cohorts we performed separate multiple logistic regressions for early loss, early plus intermediate reduction and reduction at any age, with the singleton cohort as the reference group (Table IV). All analyses showed that survivors of a vanishing co-twin were more likely to be born preterm and with low birth weight than singletons originating from a single gestation. This was even more pronounced for very preterm and very low birth weight infants, where risks were between 2 and 3 . The early vanish group had similar risk of adverse obstetric outcome as singletons from single gestations, whereas the early and intermediate cohort had significantly increased obstetric risks (Table IV).

\section{Neurological sequelae}

The incidence rates of children with cerebral palsy, neurological sequelae and neurodevelopmental diseases are reported in Table V. The crude incidence rates of children with cerebral palsy, neurological sequelae and neurodevelopmental diseases in the early (vanish $\$$ weeks), intermediate (vanish $>8$ weeks) and late (co-twin stillborn) vanish groups of the survivor cohort are listed in Table VI. There was a significant correlation between the development of neurological sequelae and neurodevelopmental diseases the later in pregnancy the spontaneous reduction occurred. No late survivor with cerebral palsy was identified and no trend could be detected according to cerebral palsy specifically (Table VI).

According to the early and intermediate vanish cohort, the incidence rates of neurological sequelae was 14.7 per 1000 $(9 / 611)$ versus 18.1 per $1000(95 / 5237)(P=0.5)$ in singletons from single gestations, and for all neurodevelopmental diseases $4.7 \%$ of the early and intermediate survivors were diagnosed versus $4.1 \%$ of the singleton cohort $(P=0.5)$. The corresponding figures for cerebral palsy was $8.2(5 / 611)$ versus 4.2 (22/5237) $(P=0.2)$, thus the incidence rate of cerebral palsy was almost twice as high in singleton survivors of an early or intermediate vanishing twin versus a singleton from a pregnancy with only one fetal heartbeat in early pregnancy. There were no significant differences between the early survivor cohort and the singleton cohort in term of cerebral palsy, neurological sequelae and neurodevelopmental diseases.

Table IV. Multiple logistic regression analyses showing predictors of preterm birth ( $<37$ weeks in upper and $<32$ weeks in lower panel) and low birth weight ( $<2500 \mathrm{~g}$ in upper and $<1500 \mathrm{~g}$ in the lower panel) in survivors of a vanishing co-twin

\begin{tabular}{|c|c|c|c|c|c|c|}
\hline Cohort & \multicolumn{3}{|c|}{ Low birth weight $(<2500 \mathrm{~g})[\mathrm{OR}(95 \% \mathrm{CI})]$} & \multicolumn{3}{|c|}{ Preterm birth (<37 weeks) [OR $(95 \% \mathrm{CI})]$} \\
\hline Spontaneous reduction & $2.0(1.5-2.6)$ & $1.7(1.2-2.2)$ & $1.3(0.9-1.9)$ & $1.6(1.2-2.0)$ & $1.3(1.0-1.7)$ & $1.1(0.8-1.5)$ \\
\hline Maternal age $=35 \mathrm{yrs}$ & $1.2(1.0-1.5)$ & $1.2(1.0-1.5)$ & $1.2(0.9-1.5)$ & $1.2(1.0-1.4)$ & $1.2(1.0-1.4)$ & $1.2(1.0-1.4)$ \\
\hline \multirow[t]{2}{*}{ ICSI } & $1.1(0.5-2.3)$ & $1.1(0.5-2.4)$ & $0.8(0.6-1.0)$ & $1.0(0.5-1.9)$ & $1.0(0.5-1.9)$ & $0.6(0.5-0.8)$ \\
\hline & All survivors & Early + intermediate reduction & Early reduction & All survivors & Early + intermediate reduction & Early reduction \\
\hline Spontaneous reduction & $3.0(1.9-4.7)$ & $2.1(1.3-3.6)$ & $1.3(0.6-2.8)$ & $3.0(1.9-4.8)$ & $2.3(1.4-4.0)$ & $1.5(0.7-3.1)$ \\
\hline Nulliparity & $1.5(0.8-2.6)$ & $1.5(0.8-2.6)$ & $1.4(0.7-2.5)$ & $1.7(0.9-3.1)$ & $1.6(0.8-2.9)$ & $1.5(0.8-2.8)$ \\
\hline $\begin{array}{l}\text { Maternal age } \geq 35 \\
\text { years }\end{array}$ & $1.6(1.1-2.3)$ & $1.6(1.1-2.5)$ & $1.5(1.0-2.4)$ & $1.6(1.0-2.4)$ & $1.5(1.0-2.3)$ & $1.5(1.0-2.4)$ \\
\hline ICSI & $0.6(0.1-4.4)$ & $0.7(0.1-4.7)$ & $0.8(0.5-1.4)$ & $0.6(0.1-4.4)$ & $0.7(0.1-4.9)$ & $0.5(0.3-1.0)$ \\
\hline
\end{tabular}

The first column represents reductions at any gestational age, the second early plus intermediate reductions and the third early loss. In all regressions, singletons from single gestations (singleton cohort) were the reference group. The independent variables entered into the analyses were spontaneous reduction versus no spontaneous reduction, parity $<1$ or $\geq 1$, maternal age $\geq 35$ years and ICSI versus IVF. Results are presented as ORs with $95 \%$ CIs. 
Table V. The number of children in the three cohorts with neurological sequelae including cerebral palsy and all children with a neuro-developmental disease

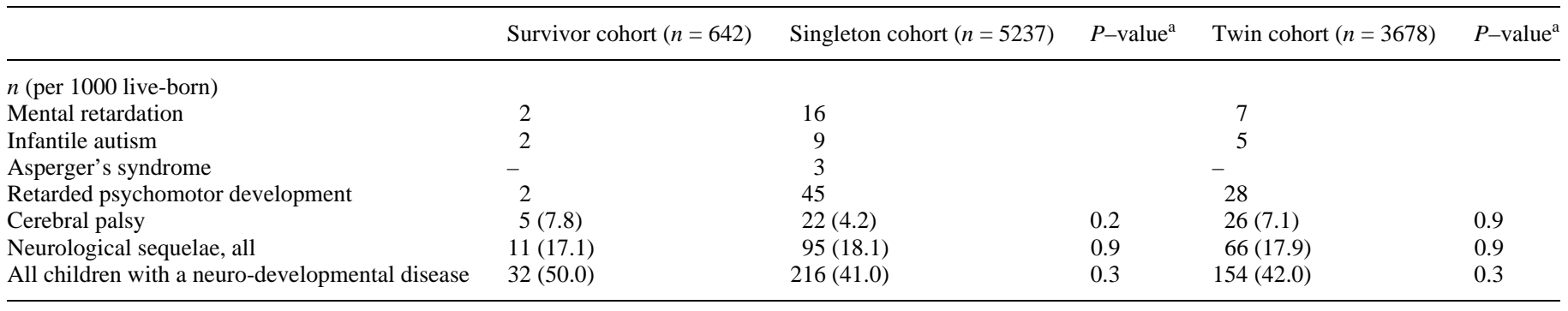

Neurological sequelae were defined as: mental retardation and severe mental developmental disturbances (DF70.0-DF79.9, DF84.0-DF89.9) including infantile autism (DF 84.0) and Asperger's syndrome (DF 84.5), cerebral palsy (DG 80.0-DG83.8) and retarded psychomotor development (DR 62.0), according to the ICD-10. aPearson's chi-square test.

Table VI. The number of children with cerebral palsy, neurological sequelae and neurodevelopmental diseases in the survivor cohort according to time of vanish of co-twin

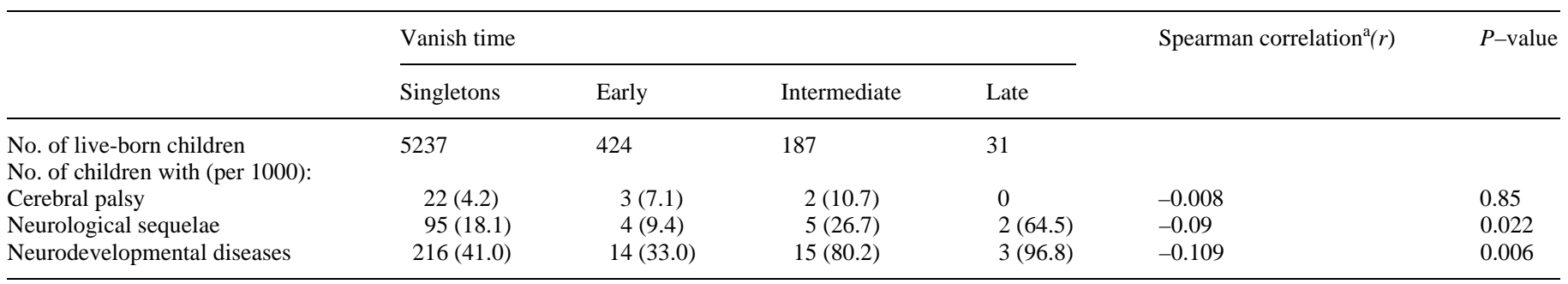

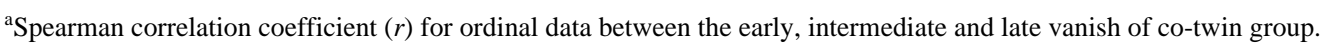

Early vanish $=$ spontaneous reduction gestational week $<8$; intermediate vanish $=$ gestational week $>8$ and $<22$; late vanish $=$ recorded as stillbirth of co-twin gestational week $>22$.

The ORs with Mantel-Haenzsel estimate after stratification for child sex and gestational age $(<32,32-36$ and $\geq 37$ weeks $)$ in the early and intermediate survivor cohort versus the singleton cohort for cerebral palsy was 1.9 (95\% CI 0.7-5.2), neurological sequelae 0.8 (95\% CI 0.4-1.6) and neurodevelopmental diseases 1.1 (95\% CI 0.7-1.7). In multiple logistic regression analysis the only significant predictive factors of neurological sequelae in the early and intermediate survivor cohort and the singleton cohort was male sex (OR 2.2; 95\% CI 1.5-3.4) and gestational age <32 weeks (OR 4.7; 95\% CI 1.7-11.4). Being an early or intermediate survivor of a vanishing twin was not a predictive factor, neither was maternal age $>35$ years, treatment method or fertility clinic. All ORs were adjusted for child age.

\section{Discussion}

About one in 10 singleton deliveries after assisted conception originate from a vanishing twin pregnancy. This study revealed a 1.7-fold increased risk of low birth weight and a 2.3 -fold increased risk of very low birth weight in these live-born survivors of a vanished co-twin compared with singletons originating from a single gestation. Moreover, the adjusted risk of child death in singleton survivors was 3.6-fold higher than in singletons from single gestations. Obstetric risks increased the later in pregnancy spontaneous reduction occurred, and were almost entirely due to spontaneous reductions at $>8$ week gestation, which accounted for one-third of the total survivor cohort. The prevalence of cerebral palsy in singleton survivors of a vanishing twin was, although not statistically significant, two-fold higher than in the singleton cohort. Furthermore, we observed a correlation between onset of vanish of co-twin and the incidence rates of neurological sequelae and neurodevelopmental diseases. Thus, spontaneous reduction has more serious consequences for the survivor the later in pregnancy the cotwin disappears. Considering that twin gestations account for $25 \%$ of all IVF pregnancies, the phenomenon of spontaneous reduction (vanishing twin) is substantial in IVF singleton deliveries compared with spontaneously conceived pregnancies. Hence, this paper provides evidence that this is one of the reasons why IVF singletons carry a higher risk of poorer outcome.

\section{Limitations of the study}

One limitation of the study is the lack of follow-up data on $3.3 \%$ of the clinical pregnancies. Emigration and patients from Greenland and the Faroe Islands, who received IVF treatment in Denmark, is the main reason for this lack of follow-up. Since registration of abortion diagnosis codes is probably less reliable than registration of deliveries, it cannot be excluded that abortions could account for a higher proportion of the missing outcome data, leading to an underestimation of spontaneous abortions. As this was equal for both study and control groups, it should not bias the study.

A second limitation is the lack of knowledge on the frequency of vanishing twins among spontaneously conceived singletons. As the IVF twin pregnancy rate is 20 -fold higher than after natural conception (one in four versus one in 80 ), it 
could be anticipated that the rate of survivors of a vanishing co-twin is also 20 -fold increased, resulting in a frequency of survivors of a vanishing co-twin of only $0.5 \%$ (10.4\% divided by 20 ) among naturally conceived singletons.

Ascertainment of spontaneous reductions is crucial to the study. Since an empty gestational sac can be difficult to visualize ultrasonically, i.e. it could be overlooked or misinterpreted as an intrauterine haematoma, there is a possibility of an underestimation of spontaneous reduction at week $<8$ in this study. Furthermore, there was a tendency for the percentage of vanishing twins to increase through the study period from 1995 to 2001 , indicating that improved ultrasound equipment and skills of the physicians or the increased focus on the importance of identification of empty gestational sacs tended to increase the rate of spontaneous reductions. The proportion of vanishing twins due to selective reduction in this study is negligible, because selective reduction on twin pregnancies to a singleton pregnancy is illegal according to the Danish law. Thus selective reduction is only performed very seldom, and only on maternal medical indication.

As benchmarks, the population-based mean birth weight of Danish IVF singletons conceived in 2003 was 3432 g versus $3517 \mathrm{~g}$ for spontaneously conceived singletons (unpublished data from the Danish National Board of Health), providing a mean birth weight difference of $85 \mathrm{~g}$. From the present study the mean birth weight in the early and intermediate survivor cohort was $3310 \mathrm{~g}$, which is considerably lower than for IVF singletons in general. Thus, some of the birth weight difference can probably be explained by spontaneous reductions. Of the $85 \mathrm{~g}$ difference in mean birth weight between IVF and spontaneously conceived singletons, vanishing twins account for $25 \%(10.4 \% \times 207 \mathrm{~g} / 85 \mathrm{~g})$. However it must be emphasized that this is without taking into account differences in maternal age and parity between naturally and assisted conceived children.

Regarding neurological sequelae and specifically cerebral palsy, another limit of the study is the relatively limited study group of 642 IVF singleton survivors of a vanishing co-twin. Since the National Danish IVF Registry was first established in 1994, the present cohort is the largest possible in Denmark, with the available data material from all public and three private fertility clinics. Furthermore, to have an appropriate follow-up age of the children of at least 30 months, at the time of retrieval of diagnoses from the National Registry of Patients, we decided not to include clinical pregnancies recorded after December 31, 2001.

However, to draw firm conclusions on the risk of neurological sequelae in IVF survivors of a vanishing co-twin, crosscountry multicentre studies with more statistical power is needed. For this purpose, a consequent registration of ultrasonographic diagnostics in first trimester is highly recommended and should be considered in the national assisted reproductive technology (ART) registries.

\section{Strengths of the study}

In 2000, $4 \%$ of Danish newborns were conceived after IVF/ ICSI (Nyboe Andersen et al., 2004). Hence, studies providing knowledge on the causes of the poorer outcome in IVF/ICSI singletons are indeed important. To our knowledge, this is the first study to assess the time-dependency of spontaneous reduction in IVF pregnancies and the only to address the longterm outcomes of these vanishing twin pregnancies. The strengths of the study were the large study cohorts and the register design. We included more than two-thirds of all ongoing IVF/ICSI singleton and twin pregnancies in Denmark in a 6 -year period and obtained data on the outcome of $97 \%$ of these pregnancies. The register design enabled us to control for potential confounders. Data on long-term morbidity on IVF children based on national registries are only provided in very few countries.

\section{Definition of neurological sequelae}

The definition of neurological sequelae and the ranking system of diagnoses was outlined in a recently published study (Pinborg et al., 2004a). To ensure an accurate recording of diagnoses, we defined neurological sequelae as only the most severe neurological and developmental disturbances. Since only one Swedish study with sufficient sample size has dealt with neurological sequelae in IVF children, we decided in our surveys to lie very close to the diagnoses applied in the Swedish paper (Strömberg et al., 2002).

\section{Comparison with other studies}

We reached similar conclusions to the first paper, which described the effect of the vanishing twin on duration of pregnancy and birth weight (Dickey et al., 2002). In 709 multiple and 5962 IVF singleton pregnancies, Dickey et al. found that pregnancy duration and birth weight was inversely related to the initial number of gestational sacs irrespective of the final birth number. They subsequently reported the effect of spontaneous reduction in exclusively IVF singleton and twins, and concluded that $15 \%$ of singleton IVF births began as a higher order gestation (Dickey et al., 2004). Additionally, in the absence of spontaneous reduction from higher order gestations, the proportion of IVF singletons born before 32 weeks was not increased compared with spontaneously conceived singletons (1.1\% versus $1.3 \%$ ). Furthermore, Lancaster (2004) showed that preterm birth occurred in $13.7 \%$ of singleton pregnancies with one gestational sac and in $18.0 \%$ with two sacs. These rates were in accordance with our results though the rates in our study were marginally lower, at $9.0 \%$ and $13.2 \%$. Moreover, a large US register study found that the risk of low birth weight increased the higher the number of fetal hearts observed on early ultrasonography for both singletons and twins (Schieve et al., 2002). Indicating that vanishing twins are not the sole contributor to the adverse outcome in IVF singletons, the same authors recently showed that elevated risk ratios of five perinatal outcomes for 3677 IVF singletons remained elevated also after restriction to pregnancies with only one fetal heart (Schieve et al., 2004). Only one study, which described no influence of vanishing twins either on gestational age or birth weight in IVF singletons, has revealed results contradictory to ours (La Sala et al., 2004). This could be due to small sample size, as only 426 singleton births were included 
and in addition only gestations with a positive heartbeat were considered.

In multifetal pregnancy reduction, Depp et al. (1996) found a statistically significant trend toward increasing frequency of intrauterine growth restriction with increasing starting fetal number, and they were the first to suggest that early implantation crowding might adversely affect surviving singletons and twins of higher order pregnancies. Our findings of higher risk of adverse obstetric outcome in the intermediate survivor group versus singletons without spontaneous reduction support the hypothesis of placental impairment as a cause of spontaneous reduction in the second trimester. The fact that the early vanish group had an equal risk to singletons without spontaneous reduction signifies that early implantation site crowding is a factor of importance to first trimester spontaneous reduction. This supports the hypothesis of a different aetiology of spontaneous reduction in first and second trimester. At present, it is not known why obstetric risk increases with increasing gestational age at onset of spontaneous reduction. A more precise subdivision of gestational age at occurrence of spontaneous reduction, which could further shed light on the problem, was not possible with the present dataset.

Another finding of the present study was that the frequency of spontaneous abortion in pregnancies with a single viable gestation in early pregnancy (6.9\%) was nearly twice the frequency in early pregnancies with two viable fetuses $(3.5 \%)$. In agreement, a recent Belgian study found that early IVF twin pregnancies have a better potential for survival of at least one fetus than singleton pregnancies (Tummers et al., 2003). However, the majority of abortions in twin gestations in our study appeared after 12 weeks of gestations and thereby at a more critical period in pregnancy than spontaneous abortion in single gestations, which primarily occurred $₫ 2$ weeks of gestation.

Only few studies have addressed the reasons for the poorer outcome in IVF versus spontaneously conceived singletons; some researchers have argued that the excess risk may be due to the underlying infertility of the couples seeking treatment rather that the treatments themselves (Pandian et al., 2001; Basso and Baird, 2003). Currently, 6\% of the Danish national birth cohort is attributable to ART and 4\% to IVF (Nyboe Andersen et al., 2004). Since the contribution of ART is rising steadily in the majority of European countries, studies on the causes of the poorer outcome in IVF singletons are highly recommended. The present study suggests poorer outcome in IVF/ICSI survivors of a vanishing co-twin compared with singletons from single gestations. As more than $10 \%$ of IVF/ICSI singletons are the result of a vanishing twin pregnancy, this may partly explain the adverse outcome in IVF/ICSI singletons. This provides us with another argument for elective single embryo transfer (eSET), and hence the impact of this study should be a step towards eSET, which will mainly reduce the high risk of twins, but apparently also the risk of spontaneous reduction and the associated adverse outcome.

\section{Acknowledgements}

We thank Steen Rasmussen and Lone Mortensen, Health Statistics, The Danish National Board of Health for their assistance in data retrieval from The Danish Medical Birth Registry and The National IVF Registry. We also thank the staff at the following fertility clinics participating in the data collection: The Fertility Clinic at Copenhagen University Hospital, Hvidovre and Herlev, Odense University Hospital, Holbaek Hospital, Aarhus University Hospital in Skejby, Braedstrup Hospital and Skive Hospital; and the following private fertility clinics: Ciconia Vest, Odense and Gentofte Fertility Clinic. The study was supported financially by grants from The Danish Medical Research Council and The Ludvig and Sara Elsass Fund. The trial was approved by the Danish Data Protection Agency (J. No. 2003-41-3611).

\section{References}

Andersen TF, Madsen M, Jorgensen J, Mellemkjaer L and Olsen J (1999) The Danish Hospital Register. A valuable source of data for modern health sciences. Dan Med Bull 46,263-268.

Basso O and Baird DD (2003) Infertility and preterm delivery, birthweight, and Caesarean section: a study within the Danish National Birth Cohort. Hum Reprod 18,2478-2484.

Depp R, Macones GA, Rosenn MF, Turzo E, Wapner RJ and Weinblatt VJ (1996) Multifetal pregnancy reduction: evaluation of fetal growth in the remaining twins. Am J Obstet Gynecol 174,1233-1238.

Dickey RP, Taylor SN, Lu PY, Sartor BM, Storment JM, Rye PH, Pelletier WD, Zender JL and Matulich EM (2002) Spontaneous reduction of multiple pregnancy: Incidence and effect on outcome. Am J Obstet Gynecol 186,77-83.

Dickey RP, Sartor BM and Pyrzak R (2004) What is the most relevant standard of success in assisted reproduction? No single outcome measure is satisfactory when evaluating success in assisted reproduction; both twin and singletons births should be counted as successes. Hum Reprod 19,783-787.

Helmerhorst FM, Perquin DAM, Donker D and Keirse MJNC (2004) Perinatal outcome of singletons and twins after assisted conception: a systematic review of controlled studies. BMJ 2004 328,261-265.

Jackson RA, Gibson KA, Wu YW and Croughan MS (2004) Perinatal outcomes in singletons following in vitro fertilization: A meta-analysis. Obstet Gynecol 103,551-563.

Lancaster PAL (2004) Number of gestational sacs and singleton IVF preterm birth. Hum Reprod 19 (Suppl 1), i85 (abstract O-245).

Landy HJ and Keith LG (1998) The vanishing twin: a review. Hum Reprod 4,177-183.

La Sala GB, Nucera G, Gallinelli A, Nicoli A, Villani MT and Blickstein I (2004) Spontaneous embryonic loss following in vitro fertilization: Incidence and effect on outcomes. Am J Obstet Gynecol 191,741-746.

Lidegaard $\varnothing$, Pinborg A and Nyboe Andersen A (2005) Imprinting diseases and IVF. Danish National IVF cohort study. Hum Reprod 20,950-954.

Munk-Jorgensen P and Mortensen PB (1997) The Danish Psychiatric Central Register. Dan Med Bull 44,82-84.

Nyboe Andersen A, Gianaroli L and Nygren KG (2004) Assisted reproductive technology in Europe, 2000. Results generated from European registers by ESHRE. Hum Reprod 19,490-503.

Pandian Z, Bhattacharya S and Templeton A (2001) Review of unexplained infertility and obstetric outcome: a 10 year review. Hum Reprod 16,2593-2597.

Pharoah POD and Adi Y (2000) Consequences of in-utero death in a twin pregnancy. Lancet 355,1597-1602.

Pinborg A, Loft A, Schmidt L, Gresien G, Rasmussen S and Nyboe Andersen A (2004a) Neurological sequelae in twins born after assisted conception: controlled national cohort study. BMJ 329,311-314.

Pinborg A, Loft A, Rasmussen S, Schmidt L, Jens Langhoff-Roos, Greisen G and Nyboe Andersen A (2004b) Neonatal outcome in a Danish national cohort of 3438 IVF/ICSI and 10362 non-IVF/ICSI twins born in 1995 to 2000. Hum Reprod 19,435-441.

Pinborg A, Loft A and Nyboe Andersen A (2004c) Neonatal outcome in a Danish national cohort of 8602 children born after in vitro fertilization (IVF) or microinsemination (ICSI): the role of twin pregnancy. Acta Obstet Gynecol Scand 83,1071-1078.

Scher AI, Petterson B, Blair E, Ellenberg JH, Grether JK, Haan E, Reddihough DS, Yeargin-Allsopp M and Nelson KB (2002) The risk of mortality or cerebral palsy in twins: a collaborative population-based study. Pediatr Res 52,671-681.

Schieve LA, Meikle SF, Ferre C, Peterson HB, Jeng G and Wilcox LS (2002) Low and very low birth weight in infants conceived with use of assisted reproductive technology. N Engl J Med 346,731-737.

Schieve LA, Ferre C, Peterson HB, Peterson HB, Macaluso M, Reynolds MA and Wright VC (2004) Perinatal outcome among singleton infants 
conceived through asssisted reproductive technology in the United States. Obstet Gynecol 103,1144-1153.

Strömberg B, Dahlquist G, Ericson A, Finnström O, Köster $M$ and Stjernquist K (2002) Neurological sequelae in children born after in-vitro fertilisation: a population based study. Lancet 359,461-465.
Tummers P, De Sutter P and Dhont M (2003) Risk of spontaneous abortion in singleton and twin pregnancies after IVF/ICSI. Hum Reprod 18,1720-1723.

Submitted on February 12, 2005; Resubmitted on May 9, 2005; Accepted on May 13, 2005 